\title{
Comparative transcriptomics of the effects of prionization and inactivation of the Swil protein in Saccharomyces cerevisiae
}

\author{
K.S. Antonets ${ }^{1,2 *}$, S.F. Kliver ${ }^{1,2}$, D.E. Polev ${ }^{1}$, A.R. Shuvalova ${ }^{1}$, E.A. Andreeva ${ }^{1,3}$, \\ S.G. Inge-Vechtomov ${ }^{1,3}$, A.A. Nizhnikov ${ }^{1,2}$ \\ ${ }^{1}$ Laboratory for Proteomics of Supra-Organismal Systems, All-Russia Research Institute for Agricultural \\ Microbiology, Pushkin, St. Petersburg, Russia \\ ${ }^{2}$ Department of Genetics and Biotechnology, St. Petersburg State University, St. Petersburg, Russia \\ ${ }^{3}$ Vavilov Institute of General Genetics RAS, St. Petersburg, Russia \\ *e-mail:kirantonez@gmail.com
}

Key words: prion, amyloid, yeast, SWI1, transcriptome

Motivation and Aims: Prions are infectious, self-perpetuating conformational states of proteins. Most part of known prions were found in yeast Saccharomyces cerevisiae. Usually, prion state is associated with the formation of protein fibrils (amyloids) and considered to be equal to functional inactivation of the protein. Swi1 is a component of the key chromatin remodeling complex SWI/SNF of yeast and was found to form prion $\left[\mathrm{SWI}^{+}\right]$. Inactivation of Swil affects many different processes in yeast cells and has the nonsense-suppression phenotype (growth on the media without adenine) in strains with the mutant variants of the SUP35 gene. The goal of this study was to compare the effects of prionization and deletional inactivation of the Swil protein on the transcription of different genes.

Methods: The next-generation RNA sequencing (RNA-seq) of the yeast transcriptomes of the $\left[\mathrm{SWI}^{+}\right],\left[s w i^{-}\right]$, and swi $1 \Delta$ strains was performed using Illumina HiSeq 2500 platform. The expression levels of several genes were analyzed using quantitative realtime PCR.

Results: Using RNA-seq we compared transcriptome-wide effects of prionization and deletional inactivation of Swil and found significant differences. In particular, about 20 yeast genes that are downregulated in the swil $\Delta$ strain, are upregulated in the $\left[S \mathrm{WI}^{+}\right]$ strain. In addition, we found that nonsense-suppression phenotype had also different mechanisms in the $\left[S W I^{+}\right]$and swil $\Delta$ strains [1]. The deletion of $S W I 1$ leads to increased expression of the ade 1-14 mutant allele, while in the $\left[\mathrm{SWI}^{+}\right]$strains nonsense-suppression is caused by downregulation of the SUP45 gene encoding eRF1 release factor.

Conclusion: Prionization of Swil protein and deletion of SWII have different effects on transcription of yeast genes and, in some cases, the consequences of prion formation are similar to "gain-of-function" mutation.

Acknowledgements: This study was supported by the Grant of the President of the Russian Federation (MK-3240.2017.4 to AAN) and the Russian Foundation for Basic Research (17-04-00816 to SGIV and 16-34-60153 to AAN). Sequencing of the transcriptomes was carried out in the Research Resource Centre "Biobank" of Research Park of SPbSU.

References

1. Antonets K.S. et al. (2017) Distinct mechanisms of phenotypic effects of inactivation and prionization of Swil protein in Saccharomyces cerevisiae. Biochemistry (Mosk.). 82(10):1147-1157. 